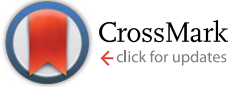

Cite this: RSC Adv., 2017, 7, 2964

Received 26th October 2016 Accepted 22nd November 2016

DOI: 10.1039/c6ra25879d

www.rsc.org/advances

\section{In situ Raman spectroscopy distinguishes between reversible and irreversible thiol modifications in L- cysteine}

\begin{abstract}
Paul Bazylewski, ${ }^{\text {a }}$ Ranjith Divigalpitiya ${ }^{\mathrm{b}}$ and Giovanni Fanchini ${ }^{\mathrm{ac}}$
In this study we introduce in situ Raman spectroscopy as an effective tool to distinguish between reversible and irreversible thiol modifications in L-cysteine, the most critical host for sulphydryls in proteins and enzymes. We place special emphasis on the conditions under which the formation and breakage of disulphide bonds is reversible and produces free thiol groups. Thiol groups from L-cysteine are highly reactive and are frequently converted into disulphide bonds via reaction with mercaptans, including $S$ methyl methanethiosulfonate (MMTS). It was previously claimed that disulphide groups can be reversibly introduced in and eliminated from L-cysteine in order to restore a free thiol group, using a reductant such as tris(2-carboxyethyl)phosphine (TCEP). Raman spectroscopy is found to effectively monitor the formation and subsequent breaking of disulphide bonds, and demonstrates that although TCEP is effective in breaking disulphide bonds, an excess of TCEP is required to reversibly form free thiol groups from L-cysteine. These results will be critical for cysteine-metal bonding investigations. Our Raman mode assignment in MMTS and TCEP also provides a benchmark for future studies using these compounds.
\end{abstract}

\section{Introduction}

L-Cysteine (Cys) is an amino acid common to many proteins and enzymes, and is of interest due to the presence of a reactive thiol group. Controlled functionalization of thiols in Cys is critical for a variety of applications including detoxification of heavy metals in living organisms, ${ }^{1}$ antioxidant capabilities of tissue and mitochondria, ${ }^{2}$ blood coagulation in mammals, ${ }^{3}$ transport across cell membranes, ${ }^{4}$ and electrochemical sensing. ${ }^{5}$ Thiol groups ( $\mathrm{SH}$ ) are present on many biologically relevant materials including glutathione and trypanotione, ${ }^{1,4}$ and cysteine-based thiols are widely employed in the formation of disulphide bonds. ${ }^{6-8}$ Disulphide bridges are important tertiary structural elements in proteins such as immunoglobulins, enzymes, hormones, procollagen and albumin. ${ }^{8}$ Disulphide bridging between cysteine residues has applications as an intermediate step in glycoprotein synthesis, ${ }^{6}$ or to reversibly control the conformation of proteins. ${ }^{9}$

Conversion of a free thiol to a disulphide bridge can be accomplished using mercaptans, a family of sulphurcontaining species that includes $S$-methyl methanethiosulfonate (MMTS). MMTS has been found to be more effective than other oxidants to form disulphides. ${ }^{10}$ Reaction of MMTS with

${ }^{a}$ Department of Physics and Astronomy, University of Western Ontario, 1151 Richmond St., London, ON N6A 3K7, Canada. E-mail: pbazylew@uwo.ca

${ }^{b} 3 M$ Canada Company, 1840 Oxford St., London, ON N5V 3R6, Canada

${ }^{c}$ Department of Chemistry, University of Western Ontario, 1151 Richmond St., London, ON N6A 5B7, Canada thiols such as cysteine residues converts the thiol group to a neutral non-hydrogen bonding methylthio group containing a disulphide bond. ${ }^{11}$ This process has been used to introduce disulphide bridges into enzymes, including ethanolamine ammonia-lyase and lactate dehydrogenase where treatment with MMTS modifies the catalytic activity. ${ }^{12,13}$ Cleaving disulphide bridges is of comparable interest in protein and enzyme studies, ${ }^{14,15}$ and can be accomplished using a variety of thiol based materials such as mercaptoethanol (b-ME), dithiothreitol (DTT), or 2-nitro-5-thiocyanatobenzoic acid (NTCB) to cleave disulphide bonds. ${ }^{\mathbf{8 1 4 - 1 6}}$ Tris(2-carboxyethyl)phosphine (TCEP) is preferred as a reducing agent in both alkaline and acidic conditions because unlike DTT, it is resistant to oxidation in air and is more hydrophilic. ${ }^{16,17}$

Although b-ME, DTT and TCEP have been used for thiol manipulation, in situ spectroscopic studies of the formation and breaking of disulphide bonds in these materials has seldom been reported. Completely reversible modification in an aqueous environment is claimed, and Raman is the ideal tool to investigate this by providing structural information while simultaneously probing for the presence of sulphur containing moieties. ${ }^{18}$ Determinations of disulphide concentration in cysteines, ${ }^{2,6,11}$ as well as the oxidation of TCEP ${ }^{14,16,17}$ have been reported, but a detailed investigation of the reversible formation and breakage of disulphide bridges in cysteines is lacking. In addition, no investigation of the vibrational structure of TCEP and MMTS has been performed, either individually or where reaction with Cys is concerned. In our study, we utilize Raman spectroscopy to study the interaction of Cys with MMTS 
and TCEP, and examine if the formation and breakage of disulphide groups is reversible and produces free thiol groups on cysteine. The Raman active modes of MMTS and TCEP are summarized, as well as those of the intermediate reaction products. We show that disulphide bonds are effectively broken by an excess of TCEP to yield free thiols.

\section{Materials and methods}

Eight cysteine-based samples were prepared in three steps according to the schematics shown in Fig. 1. In step (1), a $0.10 \mathrm{M}$ stock solution of L-cysteine (Sigma Aldrich, CAS 59-90-4) was prepared by dissolving in deionized water at room temperature, and stirring for 10 minutes. Step (2) is the reaction shown in Fig. 1(a), performed by adding $S$-methyl methanethiosulfonate (MMTS, Sigma Aldrich, CAS 2949-92-0) to the Cys : $\mathrm{H}_{2} \mathrm{O}$ solution from step (1) followed by 10 minutes of stirring at room temperature. In total three solutions of Cys : MMTS were prepared using molar ratios of $1: 0.1,1: 0.5,1: 1$. Step (3) is the reaction shown in Fig. 1(b) where tris(2-carboxyethyl)phosphine : HCl (TCEP, Sigma Aldrich, CAS 51805-45-9) was further added to the Cys : MMTS solution from step (2), followed by 10 minutes of stirring. In total 4 solutions were prepared in step (3) with Cys : TCEP molar ratios of $1: 0.1,1: 0.5,1: 1$, and $1: 2$. For Raman measurements, $3 \mathrm{~mL}$ aliquots were collected in glass vials and dried at $70{ }^{\circ} \mathrm{C}$ in air until $1 \mathrm{~mL}$ of solution remained.

Unpolarised Raman spectra were recorded in the 200-3200 $\mathrm{cm}^{-1}$ range on a Renishaw InVia Microscope equipped with a Leica DM2600M microscope and a $20 \times$ objective in a backscattering arrangement. A Renishaw $633 \mathrm{~nm}$ helium-neon laser with a maximum output power of $17 \mathrm{~mW}$ was used as the excitation source. Raman spectra were recorded using a CCD array $(1024 \times 256$ pixels $)$ that was Peltier cooled to $-70^{\circ} \mathrm{C}$, and

(a)

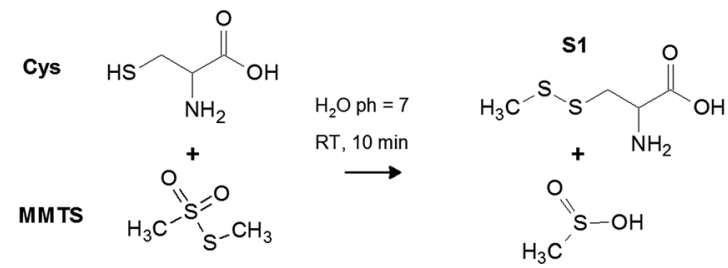

(b)

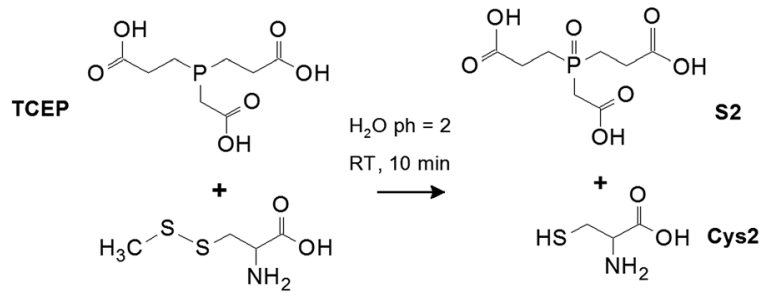

Fig. 1 (a) Schematic of L-cysteine reaction with MMTS to convert the cysteine thiol to a disulphide bond and produce product S1 (cystine). (b) Schematic of disulphide breakage to restore the thiol group of cysteine using TCEP, resulting in the irreversible oxidation of TCEP to form S2. L-Cysteine containing free thiols after disulphide breakage is labelled Cys2. equipped with a notch filter for rejection of elastically scattered

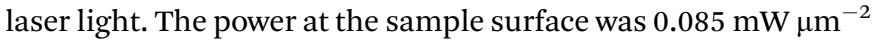
across a $20 \mu \mathrm{m}^{2}$ area. Special attention was devoted to prevent damage to the sample from the laser.

\section{Results and discussion}

\subsection{Raman modes of L-cysteine, MMTS and TCEP}

Fig. 1 shows the reaction schemes of MMTS and TCEP with Cys at room temperature. Reaction of Cys with MMTS forms product S1 containing a disulphide bond, and produces an MMTS residue. The disulphide bridge replaces the native thiol in Cys, resulting in a decrease in intensity of Cys SH stretching modes (2400-2500 $\left.\mathrm{cm}^{-1}\right)$ with a simultaneous appearance of strongly Raman active SS bending modes $\left(\sim 500 \mathrm{~cm}^{-1}\right)$ as disulphide bridges are formed. Fig. 1(b) shows the second step of the process where TCEP : $\mathrm{HCl}$ is added to the mixture to both lower the $\mathrm{pH}$ from 7 to 2 and break disulphide bonds formed by MMTS. TCEP is an effective reducing agent at mildly acidic pH, and reacts with a different mechanism of disulphide bond breakage compared to other sulfhydryl compounds such as DTT. ${ }^{16}$ The TCEP-disulphide reaction is kinetically controlled and results in the production of free thiol groups when disulphide bonds are broken. The generated thiol functional groups are less chemically reactive because ionization is suppressed by the lowered $\mathrm{pH}$, preventing the formation of a thiolate anion. ${ }^{16}$ Therefore, cross-reaction of the thiol groups and reformation of disulphide bonds should be prevented. The final step after disulphide breaking is the irreversible oxidation of TCEP to form S2 in Fig. 1(b). These reactions either individually or combined, are widely used in many applications such as synthesis of enzymes and the study of protein catalytic activity and conformation. ${ }^{4,6,9,12,13}$ However, only indirect chemical methods have been employed to examine the thiol concentration in these studies, while no direct spectroscopy measurements have been conducted to examine the reactants or the products.

Fig. 2 shows Raman spectra of Cys, MMTS, and TCEP dissolved in water. Identification of Raman active modes in these

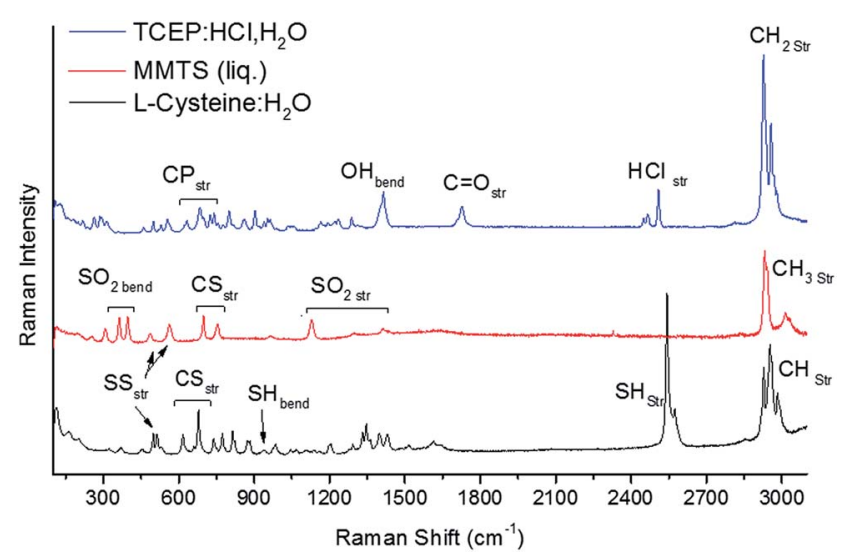

Fig. 2 Raman spectra of L-cysteine, TCEP, and MMTS. Labelled modes are used for fingerprinting of these materials and to monitor changes to sulphur-containing groups. 
reference materials provides a fingerprint of their structure. The Raman modes of Cys have been investigated in detail by Minkov et al. ${ }^{19}$ and Pawlukojc et al. ${ }^{20}$ Therefore, the Cys bands observed in Fig. 2 can be precisely assigned. From these authors, we assigned the peaks centered at $2982 \mathrm{~cm}^{-1}, 2953 \mathrm{~cm}^{-1}$ and 2928 $\mathrm{cm}^{-1}$ to $\mathrm{CH}_{2}$ asymmetric stretching, $\mathrm{CH}_{2}$ symmetric stretching and $\mathrm{CH}$ stretching, respectively. Hydrosulphide vibrations are typically located at about $2500 \mathrm{~cm}^{-1}$. From Fig. 2, it is also apparent that $\mathrm{SH}$ stretching modes in Cys present two distinct Raman active peaks, $2543 \mathrm{~cm}^{-1}$ and $2574 \mathrm{~cm}^{-1}$, which originate from $\mathrm{SH} \cdots \mathrm{O}$ and $\mathrm{SH} \cdots \mathrm{S}$ vibrations, respectively. ${ }^{20} \mathrm{SH}$ stretching vibrations are strongly Raman active and display a high intensity signal, making Raman the ideal tool for observations. Other vibrations involving sulphur are observed, including an $\mathrm{SH}$ bending mode centered at $941 \mathrm{~cm}^{-1}$, a group of CS stretching modes centered at $616 \mathrm{~cm}^{-1}, 660 \mathrm{~cm}^{-1}$ and $678 \mathrm{~cm}^{-1}$, and SS stretching modes at $498 \mathrm{~cm}^{-1}, 512 \mathrm{~cm}^{-1}$ and $529 \mathrm{~cm}^{-1}$. The lowest mode at $498 \mathrm{~cm}^{-1}$ is characteristic of cystine which is present as an impurity in the Cys sample. ${ }^{21}$ The modes at 512 $\mathrm{cm}^{-1}$ and $529 \mathrm{~cm}^{-1}$ are due to two conformers of the disulphide bond, specifically the gauche-gauche-gauche (GGG) and gauche-gauche-trans (GGT) conformers. ${ }^{22}$ These modes are expected to change drastically when the Cys thiol is modified, and can be used as a probe of any changes. Many other modes are visible in the $250-1500 \mathrm{~cm}^{-1}$ arising from other vibrations in Cys, for instance the $\mathrm{CH}_{2}$ rocking vibration $\left(775 \mathrm{~cm}^{-1}\right)$, and have been exhaustively described elsewhere. ${ }^{\mathbf{1 9 , 2 0}}$

Differently from the Raman modes of Cys, the vibrational modes of MMTS and TCEP have not been as thoroughly investigated. ${ }^{13}$ However, their assignment can be undertaken by considering what types of stretching and bending modes in these molecules involve a change in polarizability and, therefore, are Raman active. ${ }^{23}$ In MMTS, modes have been assigned as shown in Table 1 . $\mathrm{CH}$ stretching modes are found in the 2600-3200 $\mathrm{cm}^{-1}$ region. Two peak doublets are evident in this region of the MMTS spectra. They are centered at $2936 \mathrm{~cm}^{-1}$ and $3021 \mathrm{~cm}^{-1}$, respectively, and they present $\mathrm{CH}_{3}$ symmetric and asymmetric stretching modes. ${ }^{23,24}$ Below $1500 \mathrm{~cm}^{-1}, \mathrm{SO}_{2}$ stretching (1110-1430 $\mathrm{cm}^{-1}$ ) and bending (325-345 $\left.\mathrm{cm}^{-1}\right)$ vibrations can be observed, along with CS stretching modes in the $665-780 \mathrm{~cm}^{-1}$ region. ${ }^{13,24}$ These modes comprise the set of Raman active modes in liquid MMTS, and have been assigned by considering literature sources documenting the analysis of the vibrational spectra of related small molecules. ${ }^{23-25}$

The Raman-active modes of TCEP : $\mathrm{HCl}$ are also shown in Fig. 2, and were assigned according to ref. 23-25. Four peaks representing $\mathrm{CH}_{2}$ modes are visible in the $2900-3000 \mathrm{~cm}^{-1}$ region, and assigned to $\mathrm{CH}_{2}$ symmetric $\left(2927,2956 \mathrm{~cm}^{-1}\right)$ and antisymmetric stretching modes $\left(2966,2980 \mathrm{~cm}^{-1}\right)$. At lower frequencies, stretching vibrations arising from $\mathrm{HCl}$ used as a low-pH stabilizer are also visible at $2450-2520 \mathrm{~cm}^{-1}$. Vibrational peaks assigned to $\mathrm{C}=\mathrm{O}$ stretching vibrations $(1730$ $\left.\mathrm{cm}^{-1}\right)$, OH bending vibrations $\left(1415 \mathrm{~cm}^{-1}\right)$ and $\mathrm{C}-\mathrm{P}$ stretching modes (620-750 $\left.\mathrm{cm}^{-1}\right)$ are reported in Table 1 and had been previously found in relatively similar compounds in the literature. ${ }^{23-25}$ These peaks can be used as fingerprints of TCEP, particularly the $\mathrm{OH}$ bending and $\mathrm{C}=\mathrm{O}$ stretching modes, which are localized in the correspondence of carboxyl groups. A large number of additional bands are noticeable in the 250-1350 $\mathrm{cm}^{-1}$ region arising from $\mathrm{C}-\mathrm{C}$ vibrations, including: wagging, twisting, and rocking modes, and possibly others. ${ }^{23-25}$ Complex vibrations of this type are expected to be quite sensitive to molecular changes. The intensity of modes in this region drastically decreases when TCEP reacts with other compounds. For this reason, monitoring changes in the stable stretching and bending modes of sulphur containing groups has been preferred in our study.

\subsection{Disulphide bond formation via MMTS}

The formation of disulphides can be observed in detail using Raman spectroscopy to probe the vibrational modes of these bonds after reaction of Cys with MMTS and TCEP. Fig. 3 shows Raman spectra of $0.10 \mathrm{M}$ Cys : $\mathrm{H}_{2} \mathrm{O}$ after mixing with MMTS : $\mathrm{H}_{2} \mathrm{O}$ at various concentrations. MMTS reacts readily with thiol groups and the changes in modes related to sulphur can be readily monitored as the formation of a disulphide bridge significantly increases the number of sulphur related

Table 1 Raman modes of L-cysteine, MMTS and TCEP

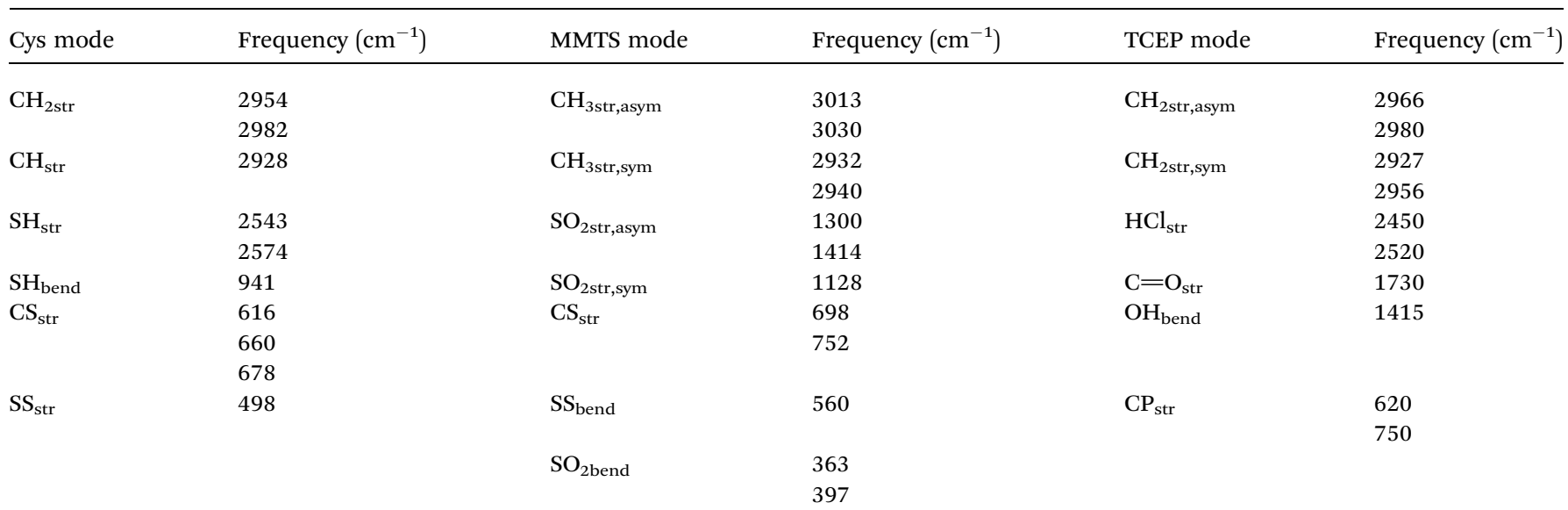



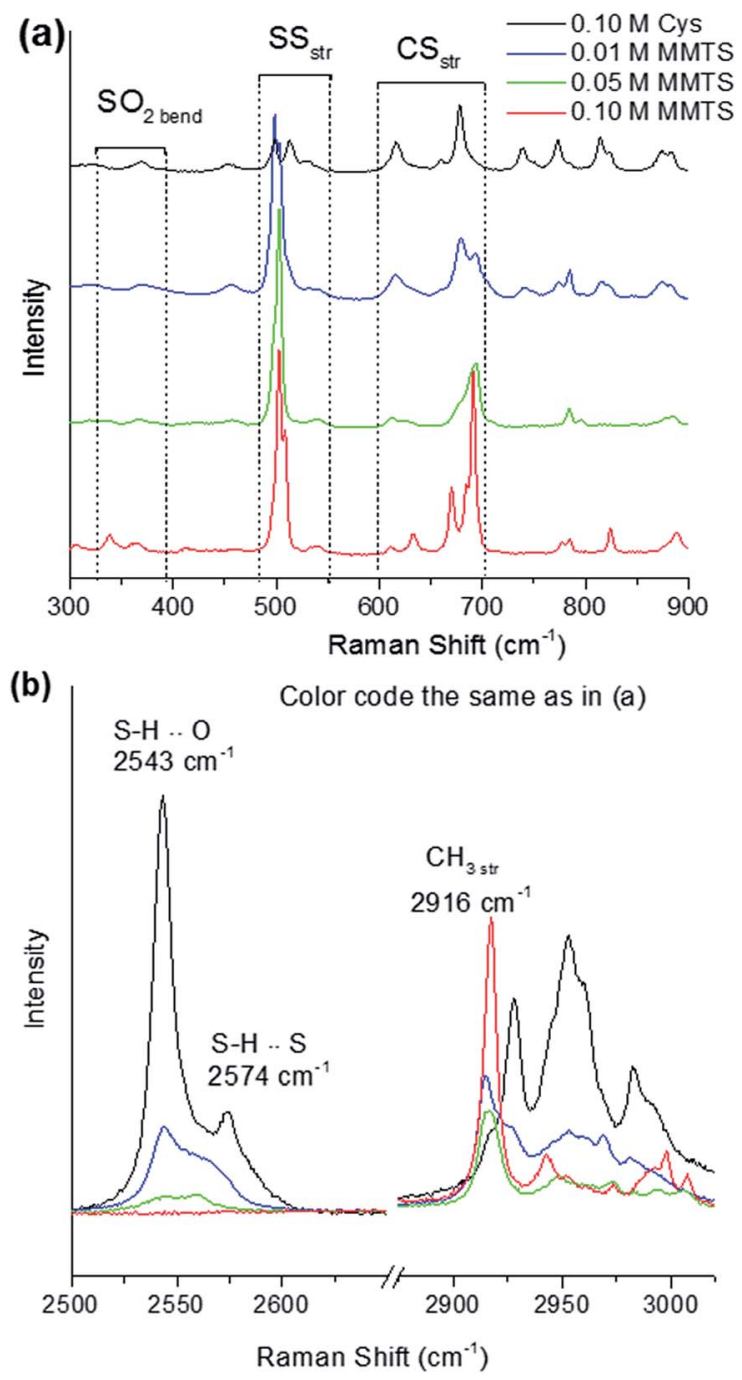

Fig. 3 Raman spectra of L-cysteine mixed with different concentrations of MMTS. (a) In the low wavenumber fingerprint region, groups of modes representing sulphur functional groups are labelled. (b) Capping of the thiol group by MMTS is observed by intensity reduction of $\mathrm{SH}$ stretching modes. $\mathrm{CH}$ stretching modes are reduced in intensity in favour of a single prominent band at $2916 \mathrm{~cm}^{-1}$ arising from stretching vibrations in the $\mathrm{CH}_{3}$ capping group, introduced by MMTS

vibrational modes in Cys. When a low concentration of $0.01 \mathrm{M}$ MMTS is combined with $0.10 \mathrm{M}$ Cys, the SH stretching mode is strongly reduced in intensity [Fig. 3(b)] in proportion to the MMTS concentration, and an equal MMTS : Cys ratio is required to reduce $\mathrm{SH}$ modes to zero intensity. In the fingerprint region below $1000 \mathrm{~cm}^{-1}$, conversion of Cys to $\mathbf{S 1}$ can be tracked through changes in CS stretching and SS bending modes. Additional bands centred at $338 \mathrm{~cm}^{-1}$ and $367 \mathrm{~cm}^{-1}$ representing $\mathrm{SO}_{2}$ bending modes are also observed, and increase in intensity proportional to MMTS concentration. From their locations, these bands cannot be assigned to unreacted MMTS. Rather, they should be assigned to residual MMTS, containing one $\mathrm{SO}_{2}$ group. The $\mathrm{SS}$ bending modes, consisting of a group of four peaks, each centred at $498 \mathrm{~cm}^{-1}, 503 \mathrm{~cm}^{-1}, 509 \mathrm{~cm}^{-1}$, and $541 \mathrm{~cm}^{-1}$ are associated with cystine, the disulphide in $\mathbf{S 1}$, and the GGG and TGT torsional conformers of these disulphides, respectively. ${ }^{21,22}$ The $498 \mathrm{~cm}^{-1}$ peak from cystine shows a maximum intensity at a low concentration of MMTS, suggesting trace amounts of MMTS promote the formation of cystine rather than product $\mathbf{S 1}$. The CS stretching mode at 660 $\mathrm{cm}^{-1}$ in unmodified Cys is red shifted to $684 \mathrm{~cm}^{-1}$, and new CS stretching modes appear at $633 \mathrm{~cm}^{-1}, 670 \mathrm{~cm}^{-1}$ and $690 \mathrm{~cm}^{-1}$ due to both the MMTS residue and the $\mathrm{S}-\mathrm{CH}_{3}$ attached to Cys to form the disulphide bridge (Table 2).

The $\mathrm{CH}_{3}$ group present in $\mathbf{S} \mathbf{1}$ also causes significant changes to $\mathrm{CH}$ stretching modes at high wavenumber from 2900-3000 $\mathrm{cm}^{-1}$. From Fig. 3(b), $\mathrm{Cys}^{\mathrm{CH}_{2}}$ modes are suppressed in favour of a strong $\mathrm{CH}_{3}$ stretching band at $2916 \mathrm{~cm}^{-1}$ in molecule $\mathbf{S 1}$. The change in structure due to the disulphide causes a shift in electron distribution into the disulphide group way from the rest of the molecule, resulting in a blue shift of $16 \pm 1 \mathrm{~cm}^{-1}$ from $2954 \mathrm{~cm}^{-1}$ in one $\mathrm{CH}_{2}$ stretching band, and a red shift of $11 \pm 1 \mathrm{~cm}^{-1}$ from $2982 \mathrm{~cm}^{-1}$ in the other. These spectra verify that MMTS quickly and efficiently produces disulphide bridges on Cys in an aqueous environment, to form product $\mathbf{S 1}$ without evidence of unreacted material. The small fraction of cystine present does not affect the reaction with MMTS. Notably, however, using trace amounts of MMTS promote the formation of cystine rather than the desired product $\mathbf{S 1}$.

\subsection{TCEP interaction with modified L-cysteine}

The disulphide bonds attached to Cys during the reaction with MMTS shown in Fig. 1(a) can be broken to result in thiol groups by mixing with TCEP. When TCEP : $\mathrm{H}_{2} \mathrm{O}$ solution $(\mathrm{pH}=1-2)$ is combined with a Cys : MMTS, a clear $\mathrm{pH}=2$ solution is obtained with no precipitation. Fig. 4(a) shows Raman spectroscopy the lower wavenumber region of several Cys : MMTS : TCEP mixtures, showing many bands characteristic of both Cys and oxidized TCEP, which are the expected products of the reaction shown in Fig. 2(b). Adding 0.01 M TCEP has a marginal effect on the vibrational spectrum, with SS bending and CS stretching

Table 2 Raman modes of reaction products S1, S2 and Cys2

\begin{tabular}{llllll}
\hline S1 mode & $\begin{array}{l}\text { Frequency } \\
\left(\mathrm{cm}^{-1}\right)\end{array}$ & S2 mode & $\begin{array}{l}\text { Frequency } \\
\left(\mathrm{cm}^{-1}\right)\end{array}$ & Cys2 mode & $\begin{array}{l}\text { Frequency } \\
\left(\mathrm{cm}^{-1}\right)\end{array}$ \\
\hline $\mathrm{CH}_{3 \text { str }}$ & 2916 & $\mathrm{CH}_{3 \text { str }}$ & 2924 & $\mathrm{SH}_{\text {str }}$ & 2460 \\
& & & 2954 & & 2530 \\
$\mathrm{CH}_{2 \text { str }}$ & 2943 & $\mathrm{C}=\mathrm{O}_{\text {str }}$ & 1725 & $\mathrm{SH}_{\text {bend }}$ & 941 \\
& 2998 & & & & \\
$\mathrm{CS}_{\text {str }}$ & 611 & $\mathrm{OH}_{\text {bend }}$ & 1411 & $\mathrm{CS}_{\text {str }}$ & 644 \\
& 633 & & & & 681 \\
& 670 & & & & \\
& 684 & & & & \\
$\mathrm{SS}_{\text {str }}$ & 690 & & & & \\
& 498 & $\mathrm{P}=\mathrm{O}_{\text {str }}$ & 1294 & & \\
& 503 & & & & \\
$\mathrm{SO}_{2 \text { bend }}$ & 509 & & & & \\
& 541 & & & &
\end{tabular}



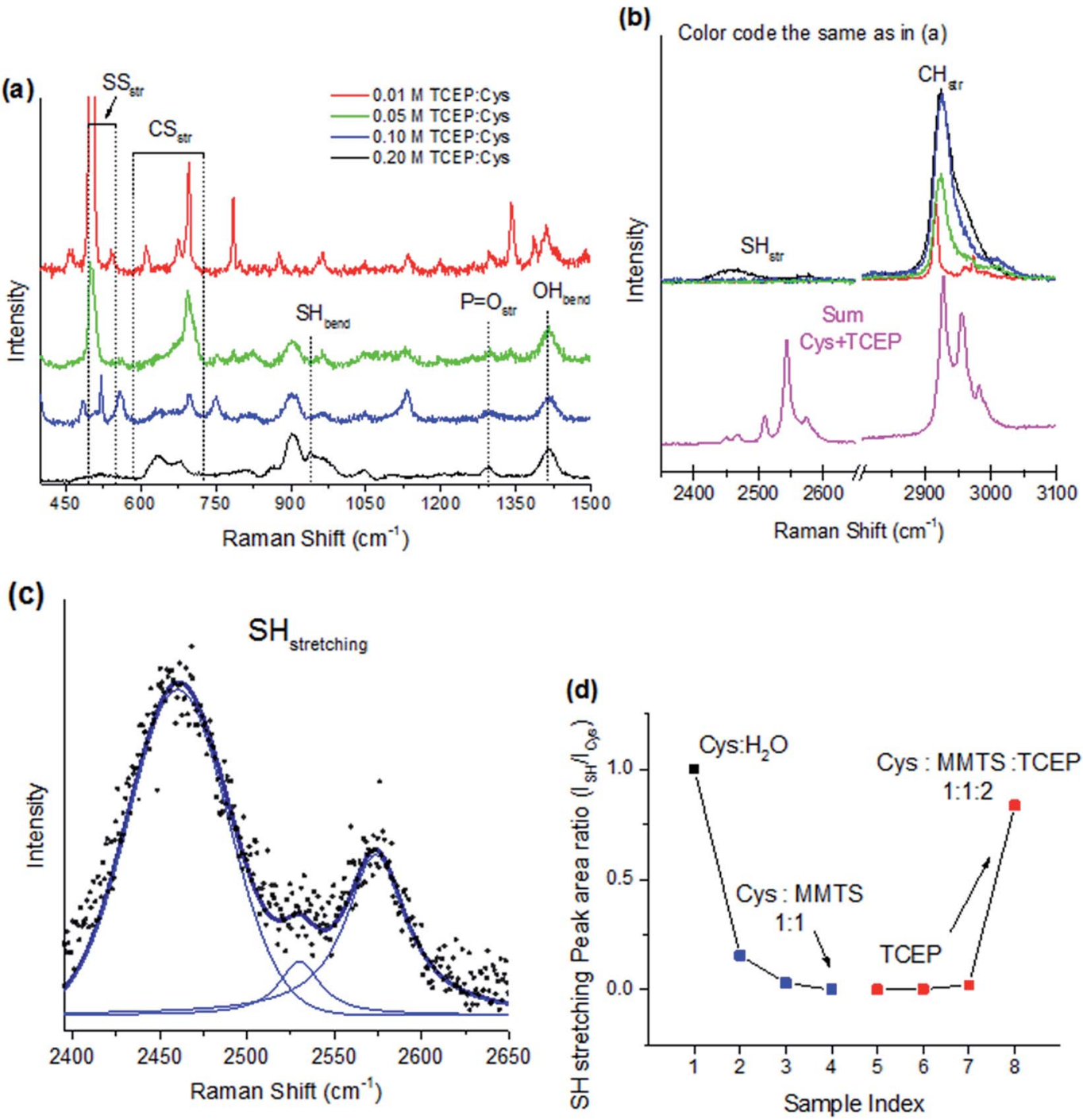

Fig. 4 Detachment of disulphide bonds with TCEP application. At equivalent concentration of L-cysteine, TCEP cleaves all disulphide bonds. (a) The lower wavenumber region displays the fingerprint of TCEP after reaction with a disulphide bond. (b) The higher wavenumber region is compared to the summation of Cys and TCEP spectra. Restoration of the Cys thiol group is observed at a Cys : TCEP ratio of $1: 2$. (c) Peak fitting using Voigt functions to characterize SH stretching modes reveals three distinct bands. (d) The integrated peak intensity for the range $2380-$ $2640 \mathrm{~cm}^{-1}$ calculated for each spectrum in Fig. 3(c) and 4(b) as a ratio relative to Cys : $\mathrm{H}_{2} \mathrm{O}$. Approximately $80 \%$ of cysteine $\mathrm{SH}$ groups are recovered with excess TCEP.

modes unchanged from the original solution of Cys : MMTS. When $0.05 \mathrm{M}$ and $0.10 \mathrm{M}$ TCEP is mixed in, new modes in the $1250-1800 \mathrm{~cm}^{-1}$ region are detected specific to the TCEP carboxyl group in the form of $\mathrm{OH}$ bending $\left(1725 \mathrm{~cm}^{-1}\right)$ and $\mathrm{C}=\mathrm{O}$ stretching modes $\left(1411 \mathrm{~cm}^{-1}\right)$. These modes are slightly blue shifted from the positions in pure TCEP due to oxidation. This is the origin of the $\mathrm{P}=\mathrm{O}$ stretching mode observed at $1294 \mathrm{~cm}^{-1}$ that is present only if TCEP is oxidized, indirectly indicating that disulphide bonds have been broken.

In the $450-750 \mathrm{~cm}^{-1}$ region, CS and SS stretching modes undergo significant changes when TCEP is mixed. The CS stretching features of compound S1, seen in Fig. 2(a), undergo profound changes at TCEP : Cys ratios of $2: 1$ or higher. The five features identified previously are present at the final stage as two broad features at 633 and $680 \mathrm{~cm}^{-1}$. The feature at 633 $\mathrm{cm}^{-1}$ is assigned to MMTS and methylthio residues from the disulphide bond breaking process. The feature at $680 \mathrm{~cm}^{-1}$ is a characteristic CS stretching mode of Cys, indicating the original structure has been recovered after treatment with excess TCEP. Changes to SS stretching modes also indicate a recovery of Cys along with the breaking of disulphide bridges. As the concentration on TCEP increases, the intensity of SS stretching modes decreases sharply. At equal molar ratios of TCEP and Cys, the disulphide band at $503 \mathrm{~cm}^{-1}$ assigned to $\mathbf{S 1}$ is reduced to zero intensity, indicating that all disulphide bonds are broken. Unquestionably these results show TCEP efficiently breaks disulphide bonds in low $\mathrm{pH}$ solutions, but the SH stretching vibrational modes must be further analysed to confirm that free thiol groups result from the breakage. 
Fig. 4(b) shows $\mathrm{CH}$ and $\mathrm{SH}$ stretching modes above 2500 $\mathrm{cm}^{-1}$ Raman shift. Changes to the $\mathrm{CH}$ stretching modes can be understood by comparison to a summation of raw Cys and TCEP spectra from Fig. 2. The line shape and peak positions of the sum correspond to their counterparts in the final solution with TCEP and Cys with the main peak positioned at $2925 \mathrm{~cm}^{-1}$. This feature is red shifted relative to the prominent $\mathrm{CH}$ stretching mode in $\mathbf{S 1}$ originating from the $-\mathrm{S}-\mathrm{CH}_{3}$ group vibrating at $2916 \mathrm{~cm}^{-1}$, verifying that $\mathbf{S 1}$ has been reacted completely with TCEP to produce unmodified Cys in the final solution. SH stretching and bending modes of unmodified Cys also become detectable when an excess of TCEP is applied. The $\mathrm{SH}$ bending mode of Cys is noticeable at $941 \mathrm{~cm}^{-1}$, and increases with increasing TCEP content, indicating that free thiols have been formed. This effect is corroborated by the presence of SH stretching modes in the $2400-2500 \mathrm{~cm}^{-1}$ region, which is demonstrated in Fig. 4(b).

Analysis of SH stretching modes is accomplished using peak fitting with Voigt functions to account for both instrumental and lifetime broadening in the $2400-2600 \mathrm{~cm}^{-1}$ region. The fitting reveals three distinct peaks centered at $2460 \mathrm{~cm}^{-1}, 2530$ $\mathrm{cm}^{-1}$, and $2573 \mathrm{~cm}^{-1}$ that are due to $\mathrm{SH} \cdots \mathrm{O}, \mathrm{SH} \cdots \mathrm{O}=\mathrm{P}$, and SH $\cdots$ S stretching vibrations, respectively. The broadening seen in these features, to be compared with the sum of the individual molecules (Fig. 4(b), magenta curve) reflects a large distribution of vibrational frequencies due increased interactions in solution, in turn causing suppression of the band intensity as it is spread across a larger wavenumber range. The $\mathrm{SH} \cdots \mathrm{O}$ band is blue shifted and suppressed in intensity relative to unmodified Cys (Fig. 2) due to interaction with oxidized TCEP in addition to water molecules. The new $\mathrm{SH} \cdots \mathrm{O}=\mathrm{P}$ band appears from coordination with oxidized TCEP, and the $\mathrm{SH} \cdots \mathrm{S}$ band is unchanged compared to Cys shown in Fig. 2. The presence of these bands and the lack of any shift in the $\mathrm{SH} \cdots \mathrm{S}$ band indicates that the Cys thiol group has been restored after reaction with TCEP.

Fig. 4(d) shows the integrated area of peaks in the 2400-2600 $\mathrm{cm}^{-1}$ region normalized to the area in Cys. The peak area decreases sharply with application of MMTS as disulphide bridges are formed. Breakage of these bonds using TCEP shows $\mathrm{SH}$ stretching mode intensity is recovered to $80 \%$ of its original value when a $2: 1$ TCEP : Cys ratio is used. Side-reactions on free thiols with oxygen to form disulphide bonds such as in cystine or reaction of TCEP with excess MMTS may account for the absence of SH stretching vibrations when lower concentrations of TCEP are used.

\section{Conclusions}

The reversible formation and cleavage of disulphide bonds on the thiol group of L-cysteine has been examined here in detail using Raman spectroscopy. MMTS was employed as an effective reactant to form a disulphide bridge in Cys. Disulphide bonds can be broken using a reducing agent, and TCEP is claimed be ideal in this application and is capable of producing free thiols on cysteines without back reactions. At a $1: 1$ ratio, MMTS is shown to completely convert all of the cysteine thiols into product $\mathbf{S 1}$ with disulphide bonds as indicated by an inverse relationship between the intensity of SH sand SS stretching modes. By contrast when a trace amount of MMTS is used, cystine is preferentially formed. TCEP was found to be effective at disulphide bond breakage, but free thiols on Cys are only evident when a molar excess of TCEP is used. Therefore, the process is found to be reversible and produce free thiols under the condition of excess TCEP.

Raman spectroscopy is used to identify the modes of two reaction products $\mathbf{S} 1$ and $\mathbf{S} 2$ in aqueous solution resulting from disulphide formation in Cys and oxidation of TCEP, respectively. These results build on the body of knowledge concerning disulphide formation in cysteines, and provide a benchmark mode assignment for Raman spectroscopy of MMTS and TCEP. Cysteines and disulphides are integral components of many protein and enzyme synthesis applications, and Raman spectroscopy has been presented as an excellent tool to probe the activity of sulphur containing groups. The use of Raman spectroscopy to monitor thiol reactions is not limited to small molecule amino acids as discussed in this work, and can be extended to the study of other small molecules, peptides, and proteins without modification. Although the number of observed Raman modes increases with molecule size, any thiol or disulphide groups present will remain highly Raman active, and can be readily observed as a probe of thiol activity.

\section{Acknowledgements}

PB acknowledges a MITACS Accelerate postdoctoral fellowship. GF acknowledges a Canada Research Chair in Carbon-based nanomaterials, financial support from the Canada Foundation for Innovation (project no. 212442), an Early Researcher Award from the Ontario Ministry of Research and Innovation, a Petro Canada Young Innovator Award and funding from the Natural Sciences and Engineering Research Council of Canada under the Discovery Grant program (grant no. RGPIN-2015-06004).

\section{Notes and references}

1 M. Enescu, J.-P. Renault, S. Pommeret, J.-C. Mialocq and S. Pin, Phys. Chem. Chem. Phys., 2003, 5, 3762-3772.

2 R. Requejo, T. R. Hurd, N. J. Costa and M. P. Murphy, FEBS J., 2010, 277, 1465-1480.

3 H. P. H. Liang, T. M. Brophy and P. J. Hogg, Biochem. J., 2011, 437, 455-460.

4 J. S. Park, S. J. Hughes, F. K. M. Cunningham and J. R. Hammond, Mol. Pharmacol., 2011, 80, 735-746.

5 S. Muralikrishna, K. Sureshkumar, T. S. Varley, D. H. Nagaraju and T. Ramakrishnappa, Anal. Methods, 2014, 6, 8698.

6 Q. Wan and S. J. Danishefsky, Angew. Chem., Int. Ed., 2007, 46, 9248-9252.

7 M. W. Pantoliano, R. C. Ladner, P. N. Bryan, M. L. Rollence, J. F. Wood and T. L. Poulos, Biochemistry, 1987, 26, 20772082.

8 R. Singh and G. M. Whitesides, Thiol-disulphide interchange, in Sulphur-Containing Functional Groups, ed. S. 
Patai and Z. Rappoport, John Wiley \& Sons, Inc., Chichester, UK, 1993, DOI: 10.1002/9780470034408.ch13.

9 M. Shimaoka, C. Lu, R. T. Palframan, U. H. von Andrian, A. McCormack, J. Takagi and T. A. Springer, Proc. Natl. Acad. Sci. U. S. A., 2001, 98, 6009-6014.

10 A. R. Karala and L. W. Ruddock, Antioxid. Redox Signaling, 2007, 9, 527-531.

11 D. Roberts, S. D. Lewis, D. P. Ballou, S. T. Olson and J. A. Shafer, Biochemistry, 1986, 25, 5595-5601.

12 M. G. Kopczynski and B. M. Babior, J. Biol. Chem., 1984, 259, 7652-7654.

13 M. E. D. Lestard, S. B. Díaz, M. E. Tuttolomondo, S. S. Cortez, M. Puiatti, A. B. Pierini and A. B. Altabef, Spectrochim. Acta, Part A, 2012, 97, 479-489.

14 J. C. Han and G. Y. Han, Anal. Biochem., 1994, 220, 5-10.

15 P. Liu, B. W. O'Mara, B. M. Warrack, W. Wu, Y. Huang, Y. Zhang, R. Zhao, M. Lin, M. S. Ackerman, P. K. Hocknell, G. Chen, L. Tao, S. Rieble, J. Wang, D. B. Wang-Iverson, A. A. Tymiak, M. J. Grace and R. J. Russell, J. Am. Soc. Mass Spectrom., 2010, 21, 837-844.

16 J. A. Burns, J. C. Butler, J. Moran and G. M. Whitesides, J. Org. Chem., 1991, 56, 2648-2650.
17 E. Burmeister Getz, M. Xiao, T. Chakrabarty, R. Cooke and P. R. Selvin, Anal. Biochem., 1999, 273, 73-80.

18 D. Pal and P. Chakrabarti, J. Biomol. Struct. Dyn., 1998, 15, 1059-1072.

19 V. S. Minkov, S. V. Goryainov, E. V. Boldyreva and C. H. Görbitz, J. Raman Spectrosc., 2010, 41, 1458-1468.

20 A. Pawlukojć, J. Leciejewicz, A. J. Ramirez-Cuesta and J. Nowicka-Scheibe, Spectrochim. Acta, Part A, 2005, 61, 2474-2481.

21 G. Zhu, X. Zhu, Q. Fan and X. Wan, Spectrochim. Acta, Part A, 2011, 78, 1187.

22 H. E. Van Wart and H. A. Scheraga, Proc. Natl. Acad. Sci. U. S. A., 1986, 83, 3064-3067.

23 M. Reichenbacher and J. Popp. Challenges in Molecular Structure Determination, Springer-Verlag, Berlin, Heidelberg, 2012, DOI: 1007/978-3-642-24390-5_2.

$24 \mathrm{~J}$. B. Lambert, Introduction to organic spectroscopy, Macmillan, New York, 1987.

25 E. Smith and G. Dent, Modern Raman Spectroscopy- A Practical. Approach, John Wiley \& Sons Ltd, England, 2005. 